\title{
Konjunkturschlaglicht
}

\section{Lieferengpässe bleiben Belastungsfaktor}

Im Verlauf des Frühjahrs und Sommers 2021 ist der zuvor sehr kräftige Aufschwung in Industrie und Welthandel weltweit ins Stocken geraten. In der Tendenz ging die Produktion leicht, der internationale Warenhandel sogar recht deutlich zurück. Maßgeblich waren Lieferengpässe und Knappheiten bei Rohstoffen, die zu stark steigenden Vorleistungspreisen und Produktionseinschränkungen auf höheren Verarbeitungsstufen geführt haben. Zu den Lieferengpässen hat beigetragen, dass die Kapazitäten im Seeverkehr seien es Schiffskapazitäten, Containerverfügbarkeiten oder Abfertigungskapazitäten in den Häfen - offenbar kaum noch in der Lage sind, zusätzliche Volumina zu bewältigen. Hierauf deutet die Entwicklung der Frachtraten hin, die sich bereits seit Ende 2020 drastisch erhöht haben. Auch bedingt durch einzelne Ereignisse von erheblicher Tragweite, wie die fast einwöchige Sperrung des Suezkanals infolge einer Schiffshavarie Ende März oder die pandemiebedingte zeitweise Schließung beträchtlicher Abfertigungskapazitäten in großen chinesischen Häfen im Sommer, hat sich der Anteil der Schiffskapazität, die zwar beladen, aber nicht in Fahrt ist, seit Beginn der Krise etwa verdoppelt - ein Indikator für das Ausmaß der Belastungen des Systems. Zwar werden die Schiffe in China seit einigen Wochen wieder ungehindert abgefertigt, dafür sind die Bestimmungshäfen nun überlastet. So beträgt die Wartezeit für Containerschiffe in den großen Häfen Kaliforniens derzeit knapp zwei Wochen. Eine spürbare Entspannung ist derzeit nicht in Sicht, sodass die weltwirtschaftliche Expansion von der logistischen Seite noch längere Zeit gebremst werden dürfte.

Darüber hinaus wird die Produktion in der Industrie dadurch behindert, dass die Produzenten von wichtigen Vor- und Zwischenprodukten der hohen Nachfrage nicht gerecht werden können. Seit längerem ist der Mangel an Halbleiterprodukten ein beschränkender Faktor, besonders sichtbar in der Automobilindustrie, wo die Produktion inzwischen weltweit stark gedrosselt wurde, in vielen Ländern auf weniger als zwei Drittel des Vorkrisenniveaus. Er dürfte noch länger bedeutsam bleiben, da die Hersteller von Computerchips inre Produktion nur langsam an die höhere Nachfrage anpassen können. Andere Engpässe, etwa bei Baumaterialien oder Chemiegrundstoffen, könnten sich rascher auflösen, sobald die Logistiksysteme wieder reibungslos funktionieren.

(c) Der/die Autor:in 2021. Open Access: Dieser Artikel wird unter der Creative Commons Namensnennung 4.0 International Lizenz veröffentlicht (creativecommons.org/licenses/by/4.0/deed.de).

Open Access wird durch die ZBW - Leibniz-Informationszentrum Wirtschaft gefördert.
Zuletzt hat eine Energiekrise in China zu zusätzlichen Problemen geführt. Eine Kombination von Anreizproblemen in der Regulierung der chinesischen Energieversorgung und umweltpolitischen Auflagen führte zu Stromknappheit und Beschränkungen für die Industrie, die ihre Produktion teilweise über Wochen deutlich senken musste. Dies hat weit über das Land hinaus Auswirkungen, auch weil der Weltmarktanteil Chinas bei der - in der Regel sehr energieintensiven - Veredelung von metallischen Rohstoffen in vielen Bereichen extrem hoch ist. Besonders gravierend ist dies im Fall von Magnesium, das insbesondere zur Herstellung von Leichtmetalllegierungen benötigt wird und zu nahezu $90 \%$ aus China kommt, aber auch Aluminium, seltene Erden oder Silizium werden in großem Umfang exportiert. Zwar hat sich die Energieversorgung in China inzwischen stabilisiert und die Produktion wurde nach jüngsten Meldungen in vielen Bereichen wieder hochgefahren. Infolgedessen haben sich auch die zwischenzeitlich zum Teil drastisch gestiegenen Preise der betroffenen Rohstoffe zuletzt wieder verringert. Im Vergleich zum Preisniveau vor der Pandemie ist jedoch nach wie vor ein deutlicher Anstieg zu verzeichnen und es ist weiterhin unsicher, inwieweit der weltweite Rohstoffbedarf in den kommenden Monaten gedeckt werden kann.

Die deutsche Wirtschaft ist in besonderem Maße von den Lieferengpässen betroffen. Im Oktober berichteten $70 \%$ der Unternehmen im Verarbeitenden Gewerbe, dass ihre Produktion durch einen Mangel an Rohstoffen oder Vormaterialien behindert wird. Im Euroraum ist der Anteil der Unternehmen, die von solchen Produktionsstörungen berichteten, zwar ebenfalls ungewöhnlich hoch, er fällt jedoch deutlich geringer als in Deutschland aus. Zu den vergleichsweise starken Beeinträchtigungen der deutschen Industrie durch die Lieferengpässe trägt bei, dass die Automobilbranche in besonderem Maße darunter leidet und diese Branche für das Verarbeitende Gewerbe - genauso wie für die gesamte Wirtschaftsleistung - eine besonders hohe Bedeutung hat (Jannsen, 2019). Während in Deutschland vor der Pandemie rund $20 \%$ der Wertschöpfung im Verarbeitenden Gewerbe vom Wirtschaftszweig Kraftwagen und Kraftwagenteile erbracht wurden, sind es in Frankreich und Italien lediglich knapp $6 \%$ und in Spanien rund $9 \%$.

Umfragen für Deutschland zeigen, dass neben der Automobilbranche auch in der Elektrotechnik mit rund $90 \%$ besonders viele Unternehmen von Produktionsstörungen aufgrund von Lieferengpässen betroffen sind (vgl. Ab- 


\section{Abbildung 1}

Produktionsbehinderungen aufgrund fehlender Vormaterialien in ausgewählten Branchen

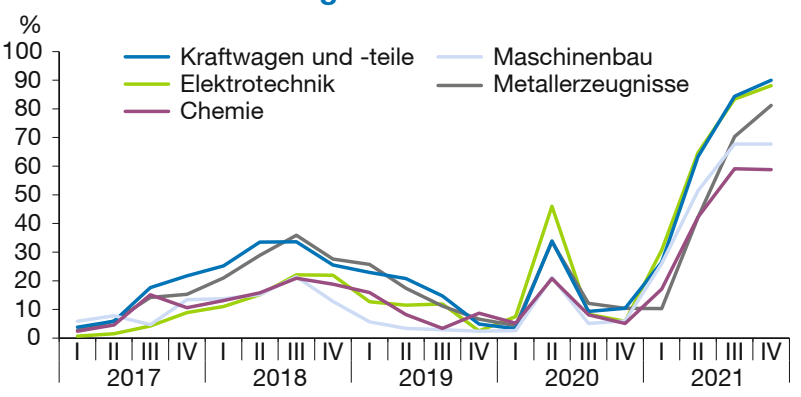

Anmerkungen: Anteil der Unternehmen, deren Produktionstätigkeit durch einen Mangel an Vorprodukten behindert wird. Wert für Q4 2021 entspricht der Oktoberumfrage.

Quelle: ifo Konjunkturumfragen Oktober 2021.

bildung 1). Freilich machen sich die Engpässe seit Jahresbeginn auch in vielen anderen Branchen zunehmend bemerkbar und der Anteil der betroffenen Unternehmen lag zuletzt auch dort vielfach bei mehr als $50 \%$. Allerdings geben diese Umfragedaten keine Auskunft darüber, in welchem Umfang die Produktion in den Unternehmen durch die Engpässe beschränkt wird. Um dies abzuschätzen, kann der historische Zusammenhang zwischen Auftragseingängen und Produktion im Verarbeitenden Gewerbe herangezogen werden (Beckmann und Jannsen, 2021). Die Differenz zwischen der tatsächlichen Produktion und dem Produktionsniveau, das angesichts der Auftragseingänge zu erwarten wäre, dürfte derzeit zu groBen Teilen auf die Lieferengpässe zurückzuführen sein.

Solche Schätzungen zeigen deutliche Unterschiede im Ausmaß der Produktionsstörungen zwischen den Branchen auf. So lag die Produktion im Wirtschaftszweig Kraftwagen und Kraftwagenteile im Dreimonatsdurchschnitt zuletzt rund $40 \%$ unterhalb des erwarteten Niveaus und damit um etwa 25 Prozentpunkte niedriger als noch im Dezember 2020 (vgl. Abbildung 2). Ebenfalls sehr kräftig - wenn auch weniger stark als in der Automobilbranche - schlagen die Lieferengpässe im Maschinenbau und in der Elektrotechnik zu Buche, wo die Produktion zuletzt rund $20 \%$ unterhalb ihres zu erwartenden Niveaus lag. Deutlich geringer sind die Produktionseinbußen demgegenüber in der chemischen und der pharmazeutischen Industrie sowie im Metallgewerbe, in denen die Produktion zuletzt nur wenig hinter den Auftragseingängen zurückhing. Im Verarbeitenden Gewerbe insgesamt haben die Auswirkungen der Lieferengpässe auf die Produktion in den vergangenen Monaten in der Tendenz noch weiter zugenommen. Im Dreimonatsdurchschnitt lag die Produktion im September um rund $16 \%$ niedriger, als es angesichts der Auftragseingänge zu erwarten gewesen wäre. Allein die seit Jahresbeginn aufgetretenen Lieferengpässe drücken das Produktionsniveau derzeit wohl um rund $10 \%$.
Abbildung 2

Auswirkungen der Lieferengpässe auf die Produktion nach Branchen

$\%$

10

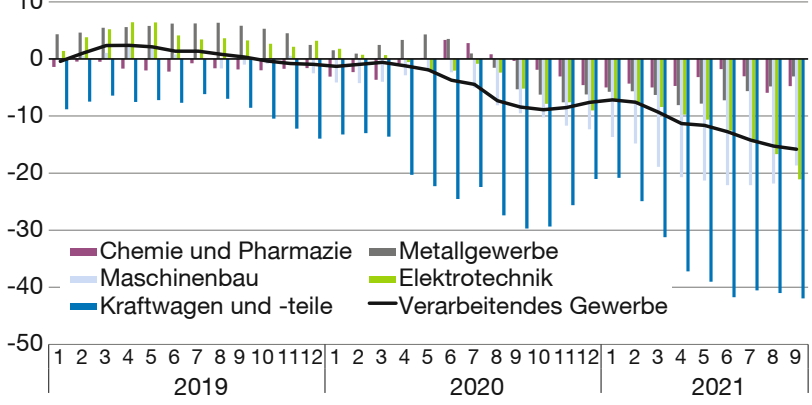

Anmerkungen: Prozentuale Abweichung der Produktion von der angesichts der Auftragseingänge zu erwartetenden Produktion. Die zu erwartende Produktion wurde durch eine Langfristbeziehung zwischen den Auftragseingängen und der Produktion geschätzt.

Quelle: Statistisches Bundesamt; eigene Berechnungen.

Aktuellen Konjunkturprognosen liegt die Annahme zugrunde, dass die Lieferengpässe im Verlauf von 2022 geringer werden und so die Produktion im Verarbeitenden Gewerbe wieder spürbar anziehen kann. Dafür spricht, dass die Unternehmen weltweit daran arbeiten, die Produktion knapper Vorprodukte auszuweiten und ihre Produktionsketten robuster auszugestalten. Auch könnte sich die Verfügbarkeit von Vorprodukten dadurch erhöhen, dass sich mit nachlassendem Einfluss von Corona auf das Wirtschaftsgeschehen die Nachfrage wieder zurück zu personennahen Dienstleistungen verlagert. Von der pandemiebedingten Sonderkonjunktur bei langlebigen Konsumgütern hatte die deutsche Industrie aufgrund ihrer Produktionsstruktur vergleichsweise wenig profitiert. Allerdings sind immer wieder neue Störungen im internationalen Wirtschaftsgefüge aufgetreten, und nicht zuletzt in Asien führen neue Corona-Fälle immer noch rasch zu spürbaren Einschränkungen der Produktion. So bleibt die Knappheit an Vorprodukten ein Unsicherheitsfaktor für die Prognose und ein Abwärtsrisiko für die Konjunktur.

Joscha Beckmann, Klaus-Jürgen Gern, Nils Jannsen nils.jannsen@ifw-kiel.de

\section{Literatur}

Beckmann, J. und N. Jannsen (2021), Lieferengpässe behindern Produktion, Wirtschaftsdienst, 101(7), 575-576, https://www.wirtschaftsdienst.eu/inhalt/jahr/2021/heft/7/beitrag/lieferengpaesse-behindernproduktion.html (5. November 2021).

Jannsen, N. (2019), Zur Bedeutung der Automobilindustrie für die deutsche Wirtschaft, Wirtschaftsdienst, 99(7), 451-456, https://www.wirtschaftsdienst.eu/inhalt/jahr/2019/heft/7/beitrag/autoindustrie-aufdem-richtigen-weg.html (5. November 2021). 